\title{
Answer to: "Histogram and Vertical Bar Diagram: Often Misapprehended Concept" by M. Lakshmanan
}

\section{Jie Du}

Published online: 13 June 2014

(C) Springer Science+Business Media New York 2014

We thank Dr Lakshmanan for his comments and agree that bar graph presentation of the data should have been referred to as such rather than as histograms. We regret this incorrect use of terms in our figure legends. With regard to the dosage of angiotensin II used in the acute study, Dr Lakshmanan may have missed it, but it was described in a paragraph under the heading "Acute Studies" of the Materials and Methods section. Finally, Dr Lakshmanan wondered why we chose to use tablet clopidogrel as the source of the drug and questioned the appropriateness of oral gavage as a delivery route. We used tablet clopidogrel for convenience as the pure chemical form of the drug was not readily accessible to us at the time of study. Clopidogrel is administrated orally in clinical treatment as it is a pro-drug and requires hepatic metabolism to get activated. It should be pointed out that we performed quality control experiment by measuring bleeding time to confirm success of oral gavage, and we note that the same delivery method was also used by other investigators in mouse studies $[1,2]$.

\section{References}

1. Liu Y, Gao XM, Fang L, Jennings NL, Su Y, Xu Q, et al. Novel role of platelets in mediating inflammatory responses and ventricular rupture or remodeling following myocardial infarction. Arterioscler Thromb Vasc Biol. 2011;31(4):834-41.

2. Hu HL, Batteux F, Chereau C, Kavian N, Marut W, Gobeaux C, et al. Clopidogrel protects from cell apoptosis and oxidative damage in a mouse model of renal ischemia-reperfusion injury. J Pathol. 2011;225: $265-75$.
J. Du ( $\bowtie)$

Beijing Anzhen Hospital, Capital Medical University,

Beijing 100029, China

e-mail: jdu@bcm.edu

J. Du

The Key Laboratory of Remodeling-related Cardiovascular Disease,

Capital Medical University, Ministry of Education, Beijing 100029,

China

J. Du

Beijing Institutes of Heart Lung \& Blood Vessel Disease,

Beijing 100029, China 